\title{
Pragmatique cognitive, argumentation et perlocution
}

Cognitive pragmatics, argumentation and perlocution

\section{Steve Oswald}

\section{(2) OpenEdition \\ 1 Journals}

\section{Electronic version}

URL: http://journals.openedition.org/aad/4793

DOI: $10.4000 /$ aad.4793

ISSN: 1565-8961

\section{Publisher}

Université de Tel-Aviv

\section{Electronic reference}

Steve Oswald, "Pragmatique cognitive, argumentation et perlocution », Argumentation et Analyse du Discours [Online], 25 | 2020, Online since 15 October 2020, connection on 17 October 2020. URL: http://journals.openedition.org/aad/4793 ; DOI : https://doi.org/10.4000/aad.4793

This text was automatically generated on 17 October 2020

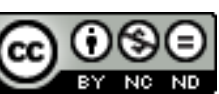

Argumentation \& analyse du discours est mis à disposition selon les termes de la licence Creative Commons Attribution - Pas d'Utilisation Commerciale - Pas de Modification 4.0 International. 


\title{
Pragmatique cognitive, argumentation et perlocution
}

\author{
Cognitive pragmatics, argumentation and perlocution
}

\section{Steve Oswald}

\section{Introduction}

1 La pragmatique contemporaine s'est développée, depuis Austin, comme l'étude du sens en contexte. Je tenterai ici d'étendre l'ensemble des phénomènes langagiers typiquement traités dans cette tradition pour y intégrer l'argumentation, une pratique communicationnelle liée au fait illocutoire qui le dépasse cependant largement par ses ramifications perlocutoires: si la compréhension du contenu d'une conclusion et de celui de ses prémisses est une étape nécessaire dans son traitement, c'est bel et bien sa portée perlocutoire - à savoir son caractère persuasif ou, plus généralement, son efficacité rhétorique - qui fait le succès de l'acte de langage argumentatif. Dans une démarche différente de celle d'Eggs, qui montre en détail comment la recherche en pragmatique devrait s'inspirer moins timidement de la rhétorique (1999), ou de celle de Jacobs, qui illustre parfaitement comment des principes pragmatiques peuvent expliquer le succès de duperies verbales (2015), je détaillerai le rôle ancillaire, mais épistémologiquement utile, que peut jouer une pragmatique cognitive de la perlocution pour doter l'analyse rhétorique d'une caution empirique (et expérimentale) supplémentaire.

2 Il s'agira d'abord (\$1) de démêler les liens complexes qui existent entre pragmatique et argumentation en tant que domaines d'étude, en passant en revue les différentes approches pragmatiques susceptibles de faire coexister les mécanismes de compréhension avec ceux qui relèvent de l'adhésion ${ }^{1}$. Puis, je présenterai les aspects de la recherche en pragmatique cognitive qui entrent en résonance avec l'étude du fait rhétorique (\$2), ce qui me permettra par la suite (\$3) d'esquisser une pragmatique cognitive de la perlocution destinée à identifier certains paramètres qui rendent une argumentation persuasive, tout en discutant ses avantages. 


\section{Pragmatique, sens, illocution et perlocution}

\subsection{Austin}

3 Partant d'une observation praxéologique du langage selon laquelle énoncer un contenu revient à accomplir un acte ayant une portée sociale (l'un des exemples canoniques d'Austin étant l'acte de baptiser), Austin est parmi les premiers - si ce n'est le premier à mettre en évidence de manière systématique la fonction performative du langage. Par là-même, il dépasse la vision vériconditionnaliste du langage qui fait de celui-ci un instrument de communication utilisé uniquement à des fins descriptives, c'est-à-dire pour rendre compte des événements ayant lieu dans le monde. Cette «illusion descriptive ", nous dit Austin, est porteuse de malentendus, mais, surtout, fait preuve de limites évidentes, tant il est vrai que le langage offre des possibilités multiples, bien au-delà de ses fonctions descriptives. «Dire, c'est faire», ce qui implique que pour rendre compte du dire, il est nécessaire d'identifier les conditions présidant au succès du faire (voir la notion de conditions de félicité chez Austin 1962 et Searle 1969). L'« acte illocutoire » est ainsi défini comme l'action que le locuteur accomplit par la formulation effective de son énoncé (promettre, requérir, demander, ordonner, asserter, conseiller, etc.).

4 La description des mécanismes de la communication réussie passe dès lors par l'étude des principes et des conventions qui gouvernent les échanges communicatifs, et qui permettent aux sujets parlants de reconnaître les actes de langage auxquels ils ont affaire. Même si les travaux de Searle ont par la suite intégré ceux de Grice en incorporant de manière plus ou moins informelle le principe de coopération de ce dernier (1989), le projet d'une théorie des actes de langage reste conventionnaliste. En effet, si les sujets parlants se comprennent lorsqu'ils communiquent, c'est qu'ils sont d'abord en mesure de reconnaitre les formes linguistiques conventionnellement associées à la performance d'actes de langage spécifiques : nous avons appris et savons, par exemple, que l'emploi du futur simple à la première personne du singulier est typiquement associé à des promesses. La pragmatique illocutoire ainsi définie par Austin se concentre sur ce niveau conventionnel du sens, dont l'étendue comprend l'étude de l'ensemble des actes de langage possibles, assortis de leurs conditions de félicité spécifiques.

5 A côté de l'acte locutoire et de l'acte illocutoire, Austin postule l'existence d'un troisième acte, l'acte perlocutoire. Il s'agit de l'acte pouvant découler de la production des deux autres, c'est-à-dire de la conséquence, dans le monde, de l'identification, par un destinataire, de l'acte illocutoire. Ainsi, nous dit Austin (1970:114), « [d]ire quelque chose provoquera souvent - le plus souvent - certains effets sur les sentiments, les pensées, les actes de l'auditoire, ou de celui qui parle, ou d'autres personnes encore. [...] Nous appellerons un tel acte un acte perlocutoire, ou une perlocution ». Le premier exemple qu'Austin nous donne de ce qu'il entend par perlocution, à mon sens très indicatif des liens que je souhaite développer ici entre comprendre et accepter (ou adhérer), est celui de la persuasion ${ }^{2}$ :

(1) Tire sur elle! ( ibid.)

6 Dans cet exemple, Austin identifie l'acte perlocutoire comme un effet de l'énoncé sur le destinataire: si l'acte de langage est heureux et que ses effets perlocutoires sont 
obtenus, alors nous pouvons dire que le locuteur, en (1), a persuadé son interlocuteur de tirer sur la personne dont il est question. Dans le modèle d'Austin, le succès de l'acte perlocutoire a pour condition nécessaire (mais non suffisante) la reconnaissance de l'acte illocutoire par le destinataire : avant d'être persuadé, un sujet parlant a besoin de comprendre le contenu de ce dont le locuteur souhaite le persuader - en d'autres termes, le succès de l'acte locutoire et celui de l'acte illocutoire sont nécessaires au succès de l'acte perlocutoire. En revanche, ce n'est pas parce que l'on comprend ce qu'un locuteur tente de nous persuader de faire que l'on est automatiquement disposé à faire ce qu'il nous dit. Comprendre n'équivaut pas à accepter. Ce dernier point me permet d'enchaîner sur les raisons qui ont poussé Austin à laisser de côté l'étude de l'acte perlocutoire. Les difficultés posées par la nature de celui-ci sont, d'après lui, rédhibitoires pour une étude systématique en parallèle à l'étude de l'acte illocutoire.

D'abord, du point de vue de sa réalité et des conditions d'émergence, il ne s'agit pas d'un acte dont le succès peut être expliqué par des principes conventionnels. S'agissant d'une conséquence extérieure à la réalisation d'un acte illocutoire, l'acte perlocutoire échappe au contrôle du locuteur. De fait, un locuteur peut accidentellement convaincre son destinataire sans avoir eu l'intention de le convaincre, tout comme il peut échouer à convaincre son destinataire alors qu'il en avait l'intention ${ }^{3}$. Prenons, pour illustrer ces deux cas de figure, la critique du film Les Huit Salopards, de Quentin Tarantino, signée Pierre Murat pour Télérama ${ }^{4}$ :

(2) «Eh bien, la violence est omniprésente dans Les Huit Salopards, elle explose,

jaillit, gicle en geysers. »

8 Faisons maintenant l'hypothèse que je supporte très mal la représentation graphique de la violence; dans un tel cas, la description que fait le journaliste va probablement m'inciter à ne pas me rendre au cinéma. Un effet perlocutoire de dissuasion est ici plausible, et cela en l'absence d'une intention dissuasive. Par ailleurs, imaginons maintenant que le journaliste, séduit par l'omniprésence de la violence, produit l'énoncé (2) précisément pour inciter ses lecteurs à aller voir l'œuvre de Tarantino. Dans ce cas, l'effet perlocutoire recherché a peu de chances de se produire chez moi, étant donné mon aversion pour la violence. Cela montre qu'un effet perlocutoire intentionné peut très bien ne pas se produire. Ce genre de considération, pour Austin, rend l'étude de la perlocution trop ardue pour en espérer une théorisation parallèle à celle de l'acte illocutoire ${ }^{5}$.

9 Ensuite, d'un point de vue formel, l'acte perlocutoire ne peut pas être préfacé par un performatif explicite; cette impossibilité est mise en avant par Austin pour asseoir la distinction entre un acte et ses conséquences. S'il est tout à fait possible de savoir si un locuteur est en train d'argumenter ou non sur la base de l'énoncé produit ${ }^{6}$, la question de savoir si ce locuteur a convaincu/persuadé est une question bien plus épineuse, que j'aborderai plus bas. Cette difficulté semble indiquer que le strict cadre linguistique est insuffisant pour le traitement de l'acte perlocutoire : Anscombre $(1980: 89)$ remarque, à juste titre, qu'alors que sa « définition de l'illocutoire - un acte accompli dans et par la parole - en fait une notion entièrement linguistique, le perlocutoire relève d'un autre ordre ", et Plantin lui emboîte le pas lorsqu'il relève les difficultés que doit surmonter le linguiste pour traiter du fait perlocutoire, qui est «traditionnellement situé hors de son champ de compétence" (1990: 48). Il en ressort, par conséquent, que l'acte perlocutoire, tel qu'il est défini par Austin, revêt une importance toute particulière : il est bel et bien une composante à part entière de l'acte communicatif, mais sa nature 
insaisissable et imprévisible en fait un objet d'étude élusif, raison pour laquelle le projet d'une pragmatique perlocutoire n'a pas véritablement abouti ${ }^{7}$.

\subsection{La pragmatique de Grice} is said) et ce qui est communiqué (speaker meaning) pour expliquer le fait que les sujets parlants comprennent des énoncés ambigus sans difficulté particulière. Pour lui, la communication réussie peut s'expliquer par l'existence d'un principe régissant les échanges d'information, qui enjoindrait les sujets parlants à coopérer en vue d'assurer un échange optimal. Ce principe de coopération s'assortit de quatre maximes conversationnelles (quantité, qualité, relation, manière), dont le respect ou l'exploitation sont instrumentaux au succès de la compréhension, et, partant, de la communication. Avec ces outils, Grice explique le succès d'échanges d'information par la reconnaissance du sens intentionnel (le fameux speaker meaning, traduit en français par vouloir-dire), ce qui témoigne de son intérêt pour l'acte illocutoire et la compréhension. La logique conversationnelle qu'il ébauche est avant tout un système de rationalité communicationnelle régi par un principe et quatre maximes dont la gestion (respect ou exploitation) par le locuteur est censée assurer un échange maximalement efficace d'information - que celle-ci soit exprimée explicitement ou implicitement ${ }^{8}$. Il convient de remarquer ici que l'idée d'un lien entre rationalité et adhésion - bien que la notion de rationalité y soit envisagée dans un sens légèrement moins spécifique que celui de Grice - est déjà présente dans la nouvelle rhétorique, comme le relève Amossy dans sa discussion éclairante sur l'apport de l'analyse du discours dans l'étude de la présentation de soi : «l'adhésion demandée est indissociable de la rationalité, seule capable d'assurer dans les affaires humaines un accord entre les hommes sur tous les sujets controversés» $(2010: 39)$. Cela ouvre en principe la voie pour discuter de la manière dont la pragmatique contemporaine, qui se donne pour mission de décrire et d'expliquer la rationalité communicative ou conversationnelle, est en mesure d'apporter quelque chose à la rhétorique - c'est du reste en partie le propos de Jacobs (2015).

11 Il est important de relever que même si les travaux de Grice concernent avant tout le niveau illocutoire du sens, ils prévoient néanmoins une extension du principe de coopération qui serait à même de couvrir le type de phénomène faisant l'objet de la présente contribution :

J'ai énoncé ces règles en supposant que le but recherché soit une efficacité maximale de l'échange d'information; cette définition est bien sûr trop étroite, et il faudrait généraliser ce schéma de règles de façon à pouvoir tenir compte de buts aussi généraux que le désir d'influencer ou de mener les autres (1979: 62, je souligne).

'est à mon sens pas un hasard si l'on retrouve dans l'exemple de l'influence une référence voilée à une problématique chère à Austin, à savoir celle de la perlocution (dont l'un des types les plus évidents est la persuasion) ${ }^{9}$. Comme Austin, Grice se pose la question de la part de la perlocution dans la communication, et, au-delà, considère que son principe de coopération doit également être en mesure d'accommoder la question, certes plus épineuse, de la coopération extralinguistique ${ }^{10}$. En ce sens, Grice appelle au développement d'une pragmatique perlocutoire, dont une partie se développera au sein d'une sous-branche de la pragmatique, à savoir la pragmatique interactionnelle.

Argumentation et Analyse du Discours, 25 | 2020 


\subsection{La pragmatique interactionnelle}

13 En suivant ici l'analyse de Catherine Kerbrat-Orecchioni (2002), il me semble pertinent de mentionner trois directions de recherche : la politesse, les figures et la présentation de soi.

14 L'étude de la politesse vise à mettre en évidence les moyens mis en œuvre par les sujets parlants pour réussir des échanges sociaux harmonieux. Les travaux fondateurs de la discipline (Lakoff 1973, Brown et Levinson 1987, Leech 1983, pour citer les plus influents) se sont appliqués à mettre en évidence le lien qui existe entre des ressources linguistiques particulières et les effets de celles-ci dans des situations d'interaction. J'emploie le terme "effet" à dessein, pour dénoter les conséquences sociales de l'interaction, conformément à la conception austinienne de la perlocution. La pragmatique des figures, quant à elle (voir par exemple Kerbrat-Orecchioni 1986, 1994, 2002), s'inscrit également de plain-pied dans une visée pragmatique perlocutoire, étant donné l'accent qu'elle met sur l'étude des différents effets de sens - également en termes d'interaction - que les figures permettent d'engendrer, en tant qu'alternatives à une formulation explicite. Finalement, les travaux en rhétorique et en analyse du discours sur la présentation de soi (voir par exemple Goffman 1982, Amossy 2010) constituent eux aussi un cadre privilégié pour l'étude de la perlocution, puisque la question de la construction de l'identité discursive - ou de l'ethos discursif - y est centrale dans la structuration des interactions verbales.

Une constante, dans ces approches qui se revendiquent pour la plupart, à raison, pragmatiques, réside dans le fait que l'étude du sens illocutoire est enrichie pour intégrer des considérations proprement perlocutoires : ce que les sujets parlants font dans la communication n'est plus seulement de l'ordre de l'identification des actes de langage, mais concerne également ce que cette identification a comme répercussions sur la situation d'interaction, sur les liens sociaux qui unissent les interlocuteurs ainsi que sur ce qui découle de cette interaction. En ce sens, la pragmatique interactionnelle intègre une dimension fonctionnelle qui lui permet de s'intéresser aux effets perlocutoires du sens, et il serait opportun de voir là une passerelle vers l'étude de l'argumentation - c'est du reste le parti pris de la pragma-dialectique, qui distingue l'acte illocutoire d'argumenter de l'acte perlocutoire de convaincre (van Eemeren et Grootendorst 1984, chapitre 3).

\subsection{Comment étudier la perlocution?}

Il ressort de ce bref panorama que la plupart des approches pragmatiques (ainsi que leurs filiations respectives) ${ }^{11}$ traitent d'une manière ou d'une autre la question de la perlocution, bien qu'aucune ne s'attelle à en formuler une théorisation explicite. Seules quelques incursions ponctuelles ont traité la question, comme celles de Marcu (2000), Gu (1993) ou Davis (1979), sans toutefois parvenir à imposer un consensus dans le domaine. Partant, il me semble opportun de réfléchir à la meilleure manière de traiter cette thématique, toujours dans un cadre pragmatique.

À mon sens, deux propriétés essentielles de l'acte perlocutoire le qualifient comme objet d'étude pertinent pour la pragmatique. D'abord, l'acte perlocutoire concerne le destinataire, puisqu'il est défini comme une conséquence de la reconnaissance d'un acte illocutoire. Cela suggère d'emblée que si l'on souhaite traiter le phénomène, une 
perspective centrée sur le destinataire semble s'imposer d'elle-même - c'est ce que fait la pragmatique cognitive contemporaine (voir Sperber et Wilson 1995, Wilson et Sperber 2012). Ensuite, le fait que l'acte perlocutoire soit une conséquence de la reconnaissance d'un acte illocutoire suggère que, même s'il n'est pas suffisant, l'acte illocutoire est susceptible de jouer un rôle dans le succès de l'acte perlocutoire. En particulier, j'aimerais explorer l'idée que les mécanismes cognitifs d'inférence permettant de calculer le sens jouent un rôle dans le succès perlocutoire. Cette posture revient à considérer qu'une théorie qui modélise l'inférence pragmatique est susceptible de nous renseigner sur les mécanismes responsables du succès de l'acte perlocutoire.

Dans ce qui suit, j'esquisse un modèle pragmatique cognitif inspiré de la théorie de la pertinence (Sperber et Wilson 1986, 1989, 1995, Wilson et Sperber 2012) destiné à traiter les aspects perlocutoires de l'argumentation.

\section{Pragmatique cognitive et argumentation}

\subsection{Pertinence, compréhension et acceptation}

Depuis Grice, la pragmatique explore régulièrement des thématiques propres à l'étude de l'argumentation : cela se voit dans les similarités entre objets d'étude. Par exemple, la notion d'inférence, au cœur de toutes les approches pragmatiques d'inspiration gricéenne, est également au cœur de toute démarche argumentative, puisque l'argumentation, dans sa vision classique, renvoie à l'articulation d'un ensemble de prémisses qui, prises ensemble, sont censées fournir une justification de la conclusion autrement dit, l'argumentation est la manifestation verbale d'un certain type d'inférence (voir aussi Oswald 2018) ${ }^{12}$. En outre, le rôle des croyances et des changements de croyance induits par la reconnaissance d'intentions communicatives (voir en particulier Sperber et Wilson 1995 sur les notions d'intention informative et communicative) est un phénomène commun aux processus de communication (comprendre le sens d'un énoncé revient à identifier l'intention du locuteur) et à ceux d'argumentation (le résultat escompté d'un argument est un effet sur le système de croyances d'un destinataire).

Une question semble naturellement émerger du rapprochement : à partir du moment où la compréhension partage un certain nombre de points communs avec la conviction/persuasion, quels liens pouvons-nous établir entre le fait de comprendre et le fait d'être convaincu? Un retour à Grice s'impose, dans la mesure où celui-ci, alors qu'il caractérise la notion de sens, mentionne explicitement l'idée de croyance : « Nous pouvons dire que 'A a voulu signifier (de manière non naturelle) quelque chose par $\mathrm{x}$ ' est grosso modo équivalent à 'A a énoncé $\mathrm{x}$ avec l'intention d'induire une croyance au moyen de la reconnaissance de cette intention » (1989:219, ma traduction, je souligne). Pour Grice, comprendre un message revient à reconnaître une intention, et cela équivaut dans les faits à entretenir une croyance au sujet de ce qui est communiqué. Grice met le doigt ici sur une idée d'apparence anodine, mais qui légitime à mon sens toute la pertinence d'un travail de recherche sur la relation entre compréhension et acceptation (définies plus haut) : la compréhension est elle-même un effet perlocutoire que l'on peut caractériser en termes d'induction de croyance. Or, nous l'avons vu, la formation de telles croyances, au terme d'un cheminement inférentiel, est également 
l'effet recherché de l'argumentation. Mais cette similarité d'effet résultant est-elle suffisante pour considérer que comprendre est un état cognitif similaire à celui d'être convaincu/persuadé?

21 Avant de pouvoir répondre à cette question, il me semble nécessaire de faire un détour par la pragmatique cognitive. Développée en réaction à la pragmatique gricéenne, mais appartenant à la même lignée inférentialiste, la théorie de la pertinence est une théorie cognitive de la communication humaine qui postule que le succès d'un échange communicatif repose non pas sur le respect de certains principes comme le principe de coopération, mais sur l'architecture cognitive de notre esprit, taillé pour traiter la pertinence de l'information. Pour Sperber et Wilson, comprendre un énoncé, c'est parvenir à établir sa pertinence contextuelle, c'est-à-dire être en mesure d'identifier en quoi il est particulièrement opportun pour l'interprète de considérer le contenu de l'énoncé dans le contexte au moment où il est communiqué.

L'un des avantages de cette théorie réside dans sa caractérisation de la notion de pertinence, une relation optimale entre l'effort cognitif nécessaire au traitement d'une information et les effets cognitifs que celle-ci peut déclencher dans l'environnement cognitif de l'individu ${ }^{13}$. La pertinence détermine si une représentation communiquée sera retenue ou rejetée dans le processus interprétatif, selon le mécanisme suivant: plus une information génère d'effets cognitifs et moins cette information demande d'efforts de traitement pour être comprise, plus elle sera jugée contextuellement pertinente, et plus elle a de chances d'être sélectionnée dans l'environnement cognitif de l'individu - et de correspondre à celle que le locuteur avait intentionnée. La compréhension de stimuli verbaux, à savoir l'identification du vouloir-dire, suit une dynamique du moindre effort doublée d'une recherche d'effets optimaux. Ainsi, pour Sperber et Wilson, le fait que les locuteurs s'efforcent de produire des énoncés contextuellement pertinents comme celui que les destinataires s'efforcent de chercher des interprétations contextuellement pertinentes sont des faits " naturels » : c'est ainsi, selon eux, que notre système cognitif fonctionne.

Deux notions cruciales, développées dans cette théorie, méritent de figurer dans cette brève présentation du cadre théorique. La première est celle de communication ostensive-inférentielle, dont les prémices étaient déjà présentes dans la caractérisation de la signification non naturelle chez Grice (1957). La communication ostensiveinférentielle est un cas d'interaction dans lequel «[1]e communicateur produit un stimulus qui rend mutuellement manifeste au communicateur et au destinataire que le communicateur veut, au moyen de ce stimulus, rendre manifeste ou plus manifeste au destinataire un ensemble d'hypothèses I " (Sperber et Wilson 1989 : 101).

La deuxième notion importante développée dans la théorie de la pertinence est celle de manifesteté (mutuelle), définie comme suit : «Un fait est manifeste à un individu à un moment donné si et seulement si cet individu est capable à ce moment-là de représenter mentalement ce fait et d'accepter sa représentation comme étant vraie ou probablement vraie» (ibid.: 65). Ainsi, les effets cognitifs sont définis en termes épistémiques : une interprétation qui correspond au vouloir-dire du locuteur est une interprétation qui permet, au prix d'un effort de traitement modulé selon l'effet escompté, d'acquérir une croyance fiable, de confirmer une croyance faiblement entretenue ou de se débarrasser d'une croyance erronée. Or cette définition ressemble à s'y méprendre à la description que l'on pourrait faire de l'adhésion. En effet, s'il est raisonnable de considérer que la compréhension est un processus au terme duquel 
l'environnement cognitif ${ }^{14}$ d'un individu a été modifié en fonction du contenu du vouloir-dire du locuteur, il est tout aussi raisonnable de décrire l'état résultant du processus de persuasion/conviction comme l'ajout, dans l'environnement cognitif du destinataire, du contenu de l'énoncé du locuteur. Comprendre la relation entre la compréhension et l'acceptation à travers une comparaison de leur état résultant ne permet par conséquent pas de les différencier clairement.

Il est donc probable que comprendre et accepter diffèrent sur les moyens mis en œuvre pour aboutir à ce même résultat. Une argumentation se présente comme une articulation de prémisses censée justifier une conclusion. En revanche, dans une communication de type informatif, le locuteur prend en charge uniquement le fait qu'il porte à l'attention du destinataire un ensemble d'hypothèses, en-dehors de tout processus de justification. La différence entre les deux cas de figure est parfois ténue, comme le remarque à juste titre Mercier (2012: 312-313) lorsqu'il discute du statut d'une information communiquée, qui peut être prise soit comme une information, soit comme un argument (à savoir comme une information censée étayer une conclusion dont le locuteur veut nous convaincre). De plus, il est également plausible de considérer qu'une même information peut être prise en charge des deux manières selon le contexte, et donc que la distinction entre un traitement argumentatif et un traitement informatif d'une hypothèse communiquée tienne à la relation que le destinataire établit entre l'information communiquée et son propre environnement cognitif.

En d'autres termes, si comprendre et accepter partagent un même résultat, les processus qui mènent à ce résultat ne sont pas les mêmes. D'après la théorie argumentative du raisonnement de Mercier et Sperber (2009, 2011, 2017), qui s'inscrit dans une vision massivement modulaire de l'esprit, cette différence se traduirait de plus au niveau cognitif par l'intervention de deux modules cognitifs différents: le module argumentatif génère et teste des arguments alors que le module de la compréhension décode et enrichit pragmatiquement l'information communiquée pour identifier le vouloir-dire. Cette distinction centrée sur la nature cognitive des processus de compréhension et d'évaluation n'exclut pas que ceux-ci puissent fonctionner de pair. C'est d'ailleurs l'hypothèse défendue dans les travaux portant sur la vigilance épistémique (Sperber et al. 2010, 2014).

\subsection{La vigilance épistémique}

Selon Sperber et ses collègues, «les humains disposent d'une série de mécanismes cognitifs de vigilance épistémique qui ciblent le risque d'être induit en erreur par autrui » (2014:21). Du point de vue évolutionniste, si la communication s'est stabilisée au sein de l'espèce humaine, c'est qu'elle est avantageuse pour celle-ci. Compte tenu du fait que la communication est susceptible d'être exploitée à des fins manipulatoires, sa stabilisation au sein de l'espèce est une indication forte qu'il doit exister des moyens de contrecarrer (voire de résister à) la tromperie et (à) la manipulation, et ce, pour qu'elle reste avantageuse. L'hypothèse de la vigilance épistémique est donc que nous sommes équipés d'une suite de mécanismes de vérification nous permettant de tester la fiabilité de l'information, sur le plan de ses sources et de ses contenus. En termes rhétoriques, nous pourrions dire que Sperber et ses collègues font l'hypothèse que l'être humain est naturellement en mesure d'évaluer l'ethos et le logos avec lesquels l'information est communiquée ${ }^{15}$. 
mes mécanismes de vérification sont déclenchés par la reconnaissance de stimuli verbaux, sur lesquels ils opèrent - tout comme les mécanismes de compréhension. La compréhension et certains mécanismes de la vigilance épistémique opèrent donc sur le même input, à savoir l'information communiquée, mais ce que chaque mécanisme en fait est différent :

La compréhension implique l'adoption d'une attitude de confiance provisoire et fragile ; cette posture mènera à l'acceptation de ce qui est compris seulement si la vigilance épistémique, qui est déclenchée par les mêmes actes communicationnels qui déclenchent la compréhension, ne fournit pas de raisons de douter (Sperber et al. $2014: 35)$.

En contexte argumentatif, la vigilance est à l'œuvre pour vérifier que la conclusion donnée découle bien des prémisses qui la soutiennent, et cela passe par l'évaluation du lien de justification ainsi que par le déploiement de processus de contrôle de fiabilité de la source d'information (par exemple en vérifiant la réputation du communicateur). De fait, il est possible de décrire la vigilance épistémique comme un ensemble de mécanismes d'évaluation critique, ceux-là même qui sont à l'œuvre lorsqu'on évalue des arguments. La particularité du traitement argumentatif d'une information (c'est-àdire de l'évaluation du lien entre les prémisses et la conclusion qui en découle), par rapport à d'autres mécanismes de vigilance épistémique, est qu'il opère sur l'output de la compréhension: avant de pouvoir déterminer si une conclusion découle des prémisses qui sont présentées, il est nécessaire de comprendre leur contenu ainsi que celui de la conclusion. Il en découle que la distinction qui nous occupe, entre comprendre et accepter, peut être décrite à l'aide de la distinction entre le traitement cognitif d'informations communiquées en tant qu'informations et le traitement cognitif d'informations communiquées en tant qu'arguments.

En résumé, la théorie de la pertinence explique comment et pourquoi nous nous comprenons (i.e., l'illocution), et la théorie de la vigilance épistémique explique comment et pourquoi nous sommes convaincus, pourquoi nous acceptons des informations (i.e., la perlocution). Je considère dès lors que l'étude des actes perlocutoires liés aux effets de l'argumentation est possible dans un cadre cognitif, en particulier un cadre cognitif pragmatique, dans la mesure où les mécanismes qui régissent le calcul du sens peuvent également influencer l'effet perlocutoire en contexte argumentatif. C'est le propos de la section suivante.

\section{Perlocution et rhétorique}

\subsection{L'efficacité rhétorique comme résultat d'une contrainte cognitive}

31 Sur un plan cognitif, dire qu'un argument a été efficace du point de vue rhétorique revient à dire (1) qu'il a résisté à une évaluation critique ou (2) qu'il a été accepté sans évaluation critique. Ces deux cas de figure à leur tour recouvrent quatre scénarios qui ont tous en commun le succès rhétorique, c'est-à-dire le fait que le contenu de l'énoncé argumentatif du locuteur, au terme du traitement cognitif opéré par le destinataire, intègre l'environnement cognitif de ce dernier :

-(A) Toutes les informations pertinentes ont été considérées et les avantages épistémiques de la conclusion prévalent.

Argumentation et Analyse du Discours, 25 | 2020 
- (B) La conclusion est immédiatement consistante avec une représentation épistémiquement forte présente dans l'environnement cognitif du destinataire.

-(C) Aucune information critique n'est présente dans l'environnement cognitif au moment de l'évaluation.

- (D) Il n'y a aucune raison de mobiliser des informations critiques. cognitif d'un destinataire a été modifié pour y intégrer la conclusion de l'argumentaire présenté par un locuteur. Cette caractérisation nous permet d'isoler deux paramètres autour desquels l'efficacité rhétorique semble s'articuler. Les cas (a) et (b) soulignent le rôle de la force épistémique : si l'argument est accepté dans ces circonstances, c'est parce que son contenu, de même que la force du lien de justification entre prémisses et conclusion, étaient épistémiquement plus forts que le contenu de toute information critique susceptible de les réfuter. Les cas (c) et (d), quant à eux, soulignent le rôle de l'accessibilité ou de la disponibilité de l'information dans le succès rhétorique: si l'argument est accepté dans ces circonstances, c'est parce que le système cognitif n'a pas considéré d'information critique, ou alors qu'il n'y a pas eu accès. Les deux paramètres qui ressortent sont susceptibles de contraindre la façon dont l'argument est évalué ; en synthétisant ces considérations, nous dirons qu'un argument est efficace du point de vue rhétorique si l'articulation entre ses prémisses et sa conclusion génère des effets cognitifs significatifs et si son coût de traitement est peu élevé.

Les paramètres identifiés correspondent aux conditions comparatives de pertinence identifiées par Sperber et Wilson $(1989: 191)$ :

- Condition comparative 1 : une hypothèse est d'autant plus pertinente dans un contexte donné que ses effets contextuels sont plus importants.

- Condition comparative 2 : une hypothèse est d'autant plus pertinente dans un contexte donné que l'effort nécessaire pour l'y traiter est moindre.

erait tentant d'objecter ici que le modèle de la pertinence est limité aux processus de compréhension, et d'arguer que ces conditions comparatives ne peuvent pas s'appliquer au traitement cognitif du matériau argumentatif. Une réponse à apporter à cette objection consiste à invoquer le principe cognitif de pertinence, selon lequel « la cognition humaine tend à la maximisation de la pertinence » (Sperber et Wilson 1995 : 260, ma traduction) : en vertu de ce principe qui caractérise le fonctionnement cognitif global, nous pouvons ainsi maintenir l'idée que la dynamique effort/effet n'est pas exclusive à la compréhension, et qu'elle s'applique à d'autres phénomènes cognitifs, parmi lesquels l'évaluation d'arguments. En ces termes, l'évaluation d'arguments est un processus cognitif qui prend comme input les représentations que les mécanismes de compréhension génèrent et qui les compare avec des représentations contenues dans l'environnement cognitif du destinataire afin de calculer quel ensemble doit être retenu (ou sélectionné), et lequel doit être rejeté. On aboutit dès lors à une description réductionniste du fait rhétorique qui a l'heur de spécifier les conditions de succès de l'acte perlocutoire : si l'argument convainc/persuade, c'est son contenu qui est retenu d'après le mécanisme évoqué plus haut. En revanche, dans le cas contraire, c'est l'ensemble d'informations critiques qui est retenu, déclenchant par là-même la nonrétention du contenu de la conclusion dans l'environnement cognitif du destinataire.

De cette discussion émerge l'idée que l'efficacité rhétorique, sur le plan perlocutoire, est le fait d'un mécanisme cognitif de "contrainte " sur l'évaluation argumentative (voir aussi Oswald 2016, Herman et Oswald 2014, et Oswald et Herman 2016). 
Cependant, nous nous devons de constater qu'il est a priori possible de postuler deux façons distinctes de contraindre une évaluation. La première consiste à contraindre l' output des processus d'évaluation, et dans ce cas on essaie d'agir sur le résultat de cette évaluation en tentant de favoriser l'acceptabilité de la thèse et de ses arguments euxmêmes dans l'environnement cognitif. Les ressources qu'un locuteur peut solliciter pour favoriser l'adhésion de cette manière forment l'éventail des arsenaux argumentatifs (Angenot 2012) et ont traditionnellement été étudiées par les théories de l'argumentation. Le deuxième moyen de contraindre l'évaluation argumentative passe par une contrainte, en amont, de l'input de cette évaluation. C'est sur cette possibilité que je vais maintenant me pencher plus en détail.

Il est possible de contraindre l'évaluation argumentative en agissant sur les représentations qui en constituent l'input, c'est-à-dire en contraignant la représentation des prémisses et des conclusions. Il s'agit, dans ce cas-là, d'interférer dans la compréhension de manière à ce que l'évaluation opère sur un input déjà biaisé. L'évaluation en tant que telle peut demeurer non-problématique et fonctionner de la manière la plus optimale qui soit, y compris selon des normes exigeantes comme les normes formelles de la déduction logique ou les normes dialectiques de la discussion critique identifiées par les pragma-dialecticiens (cf. van Eemeren et Grootendorst 2004); tant qu'elle opère sur un input biaisé, l'évaluation ne générera pas d'outputs optimaux ${ }^{16}$.

En particulier, l'input de l'évaluation argumentative peut subir des contraintes visant à (1) empêcher le destinataire, au moment où il évalue l'argument communiqué, de se représenter des informations critiques à même de mettre en péril la thèse défendue par le locuteur, ou à (2) l'amener à considérer que ce qu'il a compris est d'emblée compatible avec son environnement cognitif. Ces contraintes sur le mécanisme de compréhension visent donc à éviter que le système cognitif se représente des informations exploitables en tant que contre-arguments, et que le système ait des raisons de chercher des contre-arguments. En inscrivant notre démarche dans le cadre cognitiviste pertinentiste présenté plus haut, nous pouvons par conséquent faire l'hypothèse que deux types de stratégies sont à même de réaliser ces contraintes, chacune exploitant les paramètres d'efficacité rhétorique présentés plus haut: des stratégies d'affaiblissement et des stratégies de renforcement.

Les stratégies d'affaiblissement sont des contraintes qui agissent sur la représentation des ensembles d'information critique susceptibles de menacer l'adhésion du destinataire à la thèse du locuteur: ce sont donc des stratégies qui visent à rendre moins accessibles et épistémiquement moins forts les ensembles d'information critique. Les stratégies de renforcement, quant à elles, agissent sur les ensembles d'information qui sont compatibles avec la visée persuasive du locuteur, en les rendant plus accessibles et épistémiquement plus forts.

\subsection{Avantages}

La contribution d'un modèle comme celui qui est esquissé ici n'est pas à chercher du côté de ses vertus descriptives, dans le sens où les stratégies qu'il met à jour sont bien connues de la tradition rhétorique. Cependant, les notions d'effet cognitif et d'accessibilité de l'information évoquées plus haut résonnent avec ce que Bonhomme appelle la «saillance figurale», lorsqu'il discute de l'effet persuasif de l'hyperbole 
(2009). La caractérisation perelmanienne de l'amplification, cette figure visant à « créer la présence » (Perelman 1977 : 52), est une sorte de contrainte sur le traitement cognitif de l'information destinée à autoriser «l'accès de notre conscience à certains objets psychologiquement saillants» (Dominicy 1996: 7). L'étude de l'ethos dans le discours (par exemple chez Amossy 2010 ou chez Herman 2005), quant à elle, qui démontre le rôle quasiment moteur de l'image du locuteur dans la dynamique de l'adhésion, peut également se réinterpréter comme une contrainte épistémique dans le traitement de l'information ${ }^{17}$. L'intérêt d'un modèle cognitif comme celui qui est présenté ici est donc plutôt à chercher du côté de son pouvoir explicatif.

40 L'étude de la perlocution - qui, rappelons-le, dénote un effet sur un (ou des) individu(s) - et en particulier l'étude de la persuasion est essentiellement une question d'ordre psychologique. Expliquer pourquoi un contenu véhiculé par un énoncé finit par faire partie (ou non) de l'environnement cognitif d'un destinataire revient à comprendre les mécanismes cognitifs qui permettent de mettre à jour un environnement cognitif ${ }^{18}$. Tout l'intérêt du modèle proposé plus haut (\$3.1) est donc précisément de pouvoir déterminer, dans un cas de figure donné, quelles contraintes déclenchées par les choix linguistiques opérés par le locuteur sont susceptibles de faire mouche (voir Oswald 2014, Oswald et Herman 2016, 2020 pour des analyses empiriques de cas). La théorie de la pertinence, sur laquelle repose cette proposition, est une théorie naturaliste qui a l'avantage d'être empiriquement testable. Elle a, du reste, été testée dans des études expérimentales (voir entre autres van der Henst et Sperber 2004, ainsi que Sperber et al. 1995). Concrètement, cela signifie que les prédictions qu'elle peut faire sur un cas donné, par exemple en ce qui concerne l'identification de la représentation la plus susceptible d'être retenue dans l'environnement cognitif d'un destinataire, à contexte constant et compte tenu de différentes représentations possibles, peuvent être testées empiriquement à des fins de confirmation. In fine, l'étude du fait rhétorique peut dès lors compter sur une méthodologie expérimentale robuste destinée à valider la justesse de ses intuitions et des modèles théoriques invoqués pour l'expliquer. Il est raisonnable de conclure que cette possibilité, reposant sur une conception technique et précise de la pertinence comme un rapport entre coût de traitement et effet cognitif, représente une option intéressante à l'heure où les sciences cognitives connaissent un essor important.

41 Un modèle pragmatique cognitif de la perlocution peut trouver son utilité dans l'étude de l'argumentation de plusieurs manières. D'abord, sur un plan peut-être plus descriptif, les types d'arguments (y compris les types d'arguments fallacieux) mis au jour par la recherche en rhétorique, logique, dialectique et argumentation au sens large peuvent être réinterprétés sur le plan cognitif. Par exemple, les attaques personnelles (de type ad hominem) seront caractérisées comme des contraintes visant à affaiblir la force épistémique d'une information en décrédibilisant sa source (voir Oswald et Hart 2013). A l'inverse, on décrira les appels à l'autorité (de type ad verecundiam) comme des stratégies visant à renforcer l'information en augmentant la crédibilité d'une source (voir Oswald et Hart 2013 également). Les sophismes de distraction (traditionnellement nommés red herrings en anglais), parmi lesquels figure l'homme de paille, seront caractérisés comme des stratégies de contrôle de l'information pertinente, destinées à faire dérailler le cours de la conversation en attirant l'attention du destinataire sur des contenus qui ne sont pas ceux sur lesquels porte le désaccord, moyennant des contraintes d'effet et d'effort cognitif (voir Oswald et Lewiński 2014 et Lewiński et 
Oswald 2013). Ensuite, l'étude des figures rhétoriques peut également faire l'objet d'une telle réinterprétation et inclure, dans son traitement pragmatique cognitif, une dimension perlocutoire à même de capturer des effets comme l'efficacité rhétorique, qui serait dès lors appréhendée en termes de gestion de l'information (voir Oswald et Rihs 2014 sur la métaphore, par exemple) : après tout, le propre des figures est de proposer des alternatives à des formulations littérales qui demandent d'inférer (et donc qui contraignent la dérivation inférentielle) des contenus différents. L'avantage de cette approche est qu'elle offre un cadre fonctionnel proposant un ensemble unifié d'outils pour l'analyse de figures différentes, ce qui par extension permet d'envisager un programme cohérent destiné à capturer un ensemble pertinent de phénomènes rhétoriques. Enfin, la contribution la plus nette de ce modèle à l'étude du fait rhétorique est sans doute sa capacité à générer des validations expérimentales de l'analyse rhétorique. En ce sens, le propre du modèle présenté ici est de reconnaître l'influence du calcul du sens dans le succès de l'acte perlocutoire de conviction/de persuasion. La tâche centrale d'une telle démarche, réductionniste, nous l'avons vu, consiste donc à montrer comment le calcul du sens est en mesure de rendre saillants (c'est-à-dire accessibles et épistémiquement forts) des ensembles d'information tout en réduisant les chances que d'autres ensembles soient représentés.

\section{BIBLIOGRAPHY}

Amossy, Ruth. 2010. La présentation de soi. Ethos et identité verbale (Paris : PUF)

Anscombre, Jean-Claude. 1980. « Voulez-vous dériver avec moi ? Communications 32 : 1, 61-124

Anscombre, Jean-Claude \& Oswald Ducrot 1983. L'argumentation dans la langue (Liège : Mardaga)

Angenot, Marc. 2012. « La notion d'arsenal argumentatif : l'inventivité rhétorique dans

l'histoire », Frydman, Benoît \& Michel Meyer (éds). Chaïm Perelman. De la nouvelle rhétorique à la logique juridique (Paris : PUF), 39-68

Attardo, Salvatore. 1997. « Locutionary and perlocutionary cooperation : The perlocutionary cooperative principle », Journal of Pragmatics $27: 6,753-779$

Austin, John L. 1962. How to do things with words (Oxford : Oxford U.P.)

Austin, John L. 1970. Quand dire, c'est faire: how to do things with words (Paris : Seuil)

Bonhomme, Marc. 2009. «De l'argumentativité des figures de rhétorique », Argumentation et Analyse du Discours 2 [en ligne]

Brown, Penelope \& Steven C. Levinson. 1987. Politeness: Some Universals in Language Usage

(Cambridge : Cambridge U.P.)

Davis, Steven. 1979. «Perlocutions », Linguistics and Philosophy 3 : 2, 225-243

Dominicy, Marc. 1996. « Le genre épidictique : Une argumentation sans questionnement ?», Hoogaert, Corinne (éd.), Argumentation et questionnement (Paris : PUF), 1-12

Ducrot, Oswald. 1980. Les échelles argumentatives (Paris : Minuit) 
Eggs, Ekkehard. 1999. «Ethos aristotélicien, conviction et pragmatique moderne », Amossy, Ruth (éd.), Images de soi dans le discours. La construction de l'ethos (Lausanne : Delachaux \& Niestlé), 31-59 Goffman, Erving. 1982. Interaction ritual : essays on face-to-face behavior (New York : Pantheon) Grice, Herbert P. 1957. « Meaning », Philosophical Review $66: 3,377-388$ Grice, Herbert P. 1975. « Logic and conversation », Cole, Peter \& Jerry L. Morgan (eds.). Syntax and semantics 3: Speech acts (New York : Academic Press)

Grice, Herbert P. 1979. « Logique et conversation », Communications $30: 1,57-72$

Grice, Herbert P. 1989. Studies in the Way of Words (Cambridge, MA : Harvard U. P.)

Gu, Yueguo. 1993. « The impasse of perlocution », Journal of Pragmatics 20 : 5, 405-432

Herman, Thierry. 2005. "L'analyse de l'ethos oratoire », Lane, Philippe (éd.), Des discours aux textes: Modèles et analyses (Rouen : Publications des Universités de Rouen et du Havre), 157-182

Herman, Thierry. 2018. «Éclairages, dimension rhétorique et argumentation à l'épreuve des tweets de Donald Trump ", Argumentation et Analyse du Discours, 20 [en ligne]

Herman, Thierry \& Steve Oswald. 2014. «Introduction », Herman, Th. \& S. Oswald (éds.), Rhétorique et cognition : perspectives théoriques et stratégies persuasives (Bern : P. Lang)

Jacobs, Scott. 2015. "Les principes pragmatiques de communication dans l'argumentation ", Argumentation et Analyse du Discours 15 [en ligne]

Kerbrat-Orecchioni, Catherine. 1986. L'implicite (A. Colin)

Kerbrat-Orecchioni, Catherine. 1994. « Rhétorique et pragmatique : les figures revisitées », Langue française $101: 1,57-71$

Kerbrat-Orecchioni, Catherine. 2002. «Rhétorique et interaction », Koren, Roselyne \& Ruth Amossy (éds). Après Perelman : quelles nouvelles politiques pour les nouvelles rhétoriques ? 173-196

Lakoff, Robin. 1973. "The logic of politeness ; or, minding your P's and Q's », Corum, C., T. C. Smith-Stark \& A. Weiser (eds). Papers from the Ninth Regional Meeting of the Chicago Linguistics Society, 292-305

Leech, Geoffrey N. (1983). Principles of Pragmatics (New York \& London : Longman)

Leech, Geoffrey N. 2014. The pragmatics of politeness (Oxford \& New York : Oxford U.P.)

Lewiński, Marcin \& Steve Oswald. 2013. «When and how do we deal with straw men? A normative and cognitive pragmatic account », Maillat, Didier \& Steve Oswald (eds). Biases and constraints in communication : argumentation, persuasion and manipulation, Journal of Pragmatics 59 : B, 164-177

Lumsden, David. 2008. « Kinds of conversational cooperation », Journal of Pragmatics $40: 11,1896-$ 1908

Marcu, Daniel. 2000. « Perlocutions: The Achilles' heel of speech act theory », Journal of Pragmatics $32: 12,1719-1741$

Mercier, Hugo. 2012. « Looking for arguments », Argumentation $26: 3,305-324$

Mercier, Hugo \& Dan Sperber. 2009. « Intuitive and reflective inferences », Evans, Jonathan \& Keith Frankish (eds). In Two Minds : Dual Processes and Beyond (Oxford : Oxford U. P.)

Mercier, Hugo \& Dan Sperber. 2011. «Why do humans reason? Arguments for an argumentative theory », Behavioral and Brain Sciences $34: 2,57-74$ 
Mercier, Hugo \& Dan Sperber. 2017. The Enigma of Reason (Cambridge, MA : Harvard U. P.)

Micheli, Raphaël. 2012. « Les visées de l'argumentation et leurs corrélats langagiers : Une approche discursive », Argumentation et Analyse Du Discours 9 [en ligne]

O'Keefe, Daniel J. 2011. « Conviction, Persuasion, and Argumentation : Untangling the Ends and Means of Influence ", Argumentation $26: 1,19-32$

Oswald, Steve. (2014). « It is Easy to Miss Something you are not Looking for: A Pragmatic Account of Covert Communicative Influence for (Critical) Discourse Analysis ", Hart, Christopher \& Piotr Cap (eds.), Contemporary Studies in Critical Discourse Analysis (London: Bloomsbury), 97-119

Oswald, Steve. (2016). « Rhetoric and cognition: pragmatic constraints on argument processing ». Padilla Cruz, Manuel (ed.), Relevance Theory: Recent Developments, Current Challenges and Future Directions (Amsterdam : Benjamins), 261-285

Oswald, Steve. (2018). « Pragmatic inference and argumentative inference ». Oswald, S. \& Didier Maillat (eds.), Argumentation and Inference: Proceedings of the 2nd European Conference on Argumentation, Fribourg 2017 (Vol. 2) (London: College Publications), 615-629

Oswald, Steve. \& Christopher Hart. 2013. « Trust Based on Bias : Cognitive Constraints on Sourcerelated Fallacies », Virtues of Argumentation, 1-13

Oswald, Steve \& Thierry Herman. 2016. « Argumentation, conspiracy and the moon : A rhetoricalpragmatic analysis », Danesi, Marcel \& Sara Greco (eds.). Case studies in discourse analysis (München : Lincom), 295-330

Oswald, Steve, \& Thierry Herman. (2020). « Give the Standard Treatment of Fallacies a Chance ! Cognitive and Rhetorical Insights into Fallacy Processing », Van Eemeren, Frans H. \& Bart Garssen (eds). From Argument Schemes to Argumentative Relations in the Wild (Cham : Springer), 4162

Oswald, Steve \& Marcin Lewiński. 2014. « Pragmatics, cognitive heuristics and the straw man fallacy », Herman, Thierry \& Steve Oswald (eds). Rhetoric \& Cognition. Theoretical perspectives and persuasive strategies (Bern : P. Lang), 313-343

Oswald, Steve \& Alain Rihs. 2014. « Metaphor as argument : Rhetorical and epistemic advantages of extended metaphors ", Argumentation $28: 2,133-159$

Perelman, Chaïm.1977. L'empire rhétorique. Rhétorique et argumentation (Paris : Vrin)

Perelman, Chaïm \& Lucie Olbrechts-Tyteca. 1958. La nouvelle rhétorique : Traité de l'argumentation (Paris : PUF)

Plantin, Christian. 1990. Essais sur l'argumentation: Introduction linguistique à l'étude de la parole argumentative (Paris : Kimé)

Searle, John R. (1969). Speech Acts: An Essay in the Philosophy of Language (Cambridge: Cambridge U. P.)

Sperber, Dan, Francesco Cara \& Vittorio Girotto. 1995. « Relevance theory explains the selection task », Cognition $57: 1,31-95$

Sperber, Dan, Fabrice Clément, Christophe Heintz, Deirdre Wilson et al. 2010. « Epistemic vigilance ", Mind and Language 25 : 4, 359-393

Sperber, Dan, Fabrice Clément, Christophe Heintz, Deirdre Wilson et al. 2014. «La vigilance épistémique ", Herman, Thierry \& Steve Oswald (éds). Rhétorique et cognition : perspectives théoriques et stratégies persuasives (Bern : P. Lang), 21-67 
Sperber, Dan \& Deirdre Wilson. 1995 [1986]. Relevance. Communication and cognition (Oxford : Blackwell)

Van der Henst, Jean-Baptiste \& Dan Sperber. 2004. « Testing the Cognitive and Communicative Principles of Relevance », Noveck, Ira A. \& D. Sperber (eds). Experimental Pragmatics (London : Palgrave Macmillan),141-171

Van Eemeren, Frans H. \& Rob Grootendorst. 1984. Speech acts in argumentative discussions: A theoretical model for the analysis of discussions directed towards solving conflicts of opinion (Dordrecht : Foris)

Van Eemeren, Frans H., \& Rob Grootendorst. 2004. A Systematic Theory of Argumentation: The Pragma-dialectical Approach (Cambridge : Cambridge U. P.)

Wilson, Deirdre \& Dan Sperber. 2012. Meaning and relevance (Cambridge : Cambridge U. P.)

\section{NOTES}

1. Faisant écho à la notion d'adhésion telle qu'elle est envisagée par Perelman et OlbrechtsTyteca (1958), je m'intéresse donc ici aux conséquences, sur le plan cognitif, d'une démarche de conviction ou de persuasion.

2. Je remercie un.e relecteur.trice m'ayant donné l'opportunité de clarifier mon emploi de la distinction entre comprendre et accepter. Je définis la compréhension en termes représentationnels - comprendre un énoncé signifie être en mesure d'identifier le vouloir-dire du locuteur, i.e., de se le représenter - et l'acceptation en termes épistémiques - accepter un énoncé signifie tenir pour vrai, approprié, légitime, etc. son contenu. Ainsi, il est tout à fait possible de comprendre le contenu d'un énoncé sans l'accepter ( «a terre est plate » est parfaitement intelligible pour moi, même si je pense que c'est factuellement faux, de la même manière que je comprends le sens de la requête de mon fils de 4 ans lorsqu'il me dit «Papa, achète-moi un cheval», sans pour autant accéder à celle-ci et m'engager dans une telle acquisition); il est en revanche moins aisé d'accepter quelque chose (la vérité d'une proposition, la requête d'autrui) sans comprendre le vouloir-dire du locuteur (si mon fils de 4 ans me dit « Papá, dame 100 francos ", et que je ne parle pas espagnol, je ne serai pas en mesure de comprendre qu'il me demande 100 francs).

3. On ne trouve pas de distinction chez Austin entre convaincre et persuader. Mon propos suit cette ligne, mais reconnaît la pertinence d'une telle distinction, notamment pour distinguer les moyens mis en œuvre pour remporter l'adhésion de l'adhésion elle-même. Il sera question ici de l'effet d'un énoncé sur l'environnement cognitif d'un destinataire ; en ce sens, je considérerai que la conviction et la persuasion partagent pour état résultant le gain de l'adhésion du destinataire (voir également O’Keefe 2012).

4. Disponible ici : https://bit.ly/38lLsUg (consulté le 7 juillet 2020).

5. Notons au passage que dans les deux cas considérés l'échec de l'acte perlocutoire survient suite à la réussite de l'acte illocutoire. Cela atteste une fois de plus du lien nécessaire mais non suffisant qui existe entre les deux.

6. De nombreuses approches de l'argumentation s'alignant sur la théorie pragma-dialectique (voir par exemple van Eemeren et Grootendorst 2004) adoptent opportunément une conception illocutoire de l'argumentation, qu'elles considèrent comme un acte de langage complexe.

7. Voir également la discussion de Marcu (2000) à ce propos.

8. Toute la force du modèle gricéen réside dans le fait qu'il capture le sens explicite et le sens implicite de manière systématique. 
9. On trouve, dans le paradigme des actes perlocutoires liés à l'influence, ses différentes variantes (persuasion, manipulation, coercition, etc.) mais également des actes à d'autres visées, comme l'insinuation, l'insulte, la menace, ou encore la plaisanterie (la liste demeure ouverte). Tous partagent le fait que leur succès n'est pas nécessairement garanti par leur reconnaissance en tant que tel ; en outre, tous ces actes ne peuvent être préfacés d'un verbe performatif explicite (voir également la discussion d'Anscombre 1980).

10. Voir à ce sujet les travaux d'Attardo (1997) sur un principe de coopération perlocutoire, et l'article de Lumsden (2008) pour une synthèse de la question.

11. Bien que je ne la discute pas en détail, j'inclus également la pragmatique intégrée inspirée par Ducrot dans ce bref panorama, vu que l'intérêt pour la dimension argumentative du langage de cette approche peut évidemment être relié à la question de la perlocution.

12. Cette vision classique sous-tend aussi bien la "visée argumentative " d'Amossy, la "visée justificatoire " de Micheli (2012), et plus généralement la séquence argumentative typique d'approches toulminiennes. Elle est également présente dans des approches normatives en logique formelle et informelle, qui interrogent ce qui fonde l'acceptabilité de l'inférence argumentative.

13. Les effets cognitifs sont généralement décrits comme l'acquisition d'une nouvelle croyance, le renforcement d'une croyance faiblement entretenue ou la suppression d'une croyance qui s'avère fausse.

14. L'environnement cognitif est défini dans la théorie de la pertinence comme l'ensemble des faits qui sont manifestes à un individu (ce que l'individu sait, ce qu'il peut savoir et l'information à laquelle il peut avoir accès).

15. On trouve cette idée, exprimée différemment, dans certains travaux de Danblon (par exemple 2014) et d'Eggs (1999). La particularité de l'approche cognitive présentée ici tient au fait que les phénomènes reliés à l'ethos et au logos, dans ce cadre, sont traités dans une perspective naturaliste soutenue par un paradigme expérimental (voir Sperber et al. 2014 pour un panorama complet).

16. Pour prendre une analogie culinaire, malgré un respect scrupuleux de la meilleure recette de cake, le résultat de la préparation sera décevant, pour ne pas dire immangeable, si l'on utilise du sable en lieu et place de farine.

17. Pour simplifier, nous dirons que l'ethos d'un locuteur, qui lui confère sa crédibilité auprès de son auditoire, peut élever le statut épistémique des énoncés qu'il profère. Les dynamiques de construction de l'image de soi permettent somme toute de transférer la légitimité d'un orateur vers celle de son propos, et par extension vers son statut épistémique.

18. Bien évidemment, je ne nie pas que des paramètres sociaux, culturels, institutionnels et situationnels jouent un rôle important dans toute entreprise de persuasion. Nonobstant, je fais l'hypothèse qu'ils peuvent trouver leur place dans la modélisation d'un processus cognitif en tant que paramètres dont la présence (ou l'absence) et la qualité sont susceptibles d'influencer le résultat escompté par le locuteur.

\section{ABSTRACTS}

This paper discusses the links between pragmatics (defined as the study of meaning in context) and the study of argumentation in order to ground a pragmatic approach to perlocution. Traditionally devoted to the study of illocutionary meaning, in the vein of speech act theory, 
pragmatics has tended to exclude the perlocutionary act from the set of phenomena it set out to account for. Here I try to show how different pragmatic approaches over time have kept some space to account for the latter and resort to cognitive pragmatics to defend the claim that this particular vein of pragmatic research provides tools for a pragmatic account of persuasion/ conviction, which is, by essence, a perlocutionary act. The methodological advantages of this proposal, which will be of interest to rhetoricians, are also discussed.

Cet article examine les liens qui existent entre la pragmatique (définie comme l'étude du sens en contexte) et l'étude de l'argumentation pour poser les bases d'une pragmatique de la perlocution. Traditionnellement dévolue à l'étude du sens illocutoire, dans la lignée de la théorie des actes de langage, la pragmatique a longtemps exclu l'acte perlocutoire de l'ensemble des phénomènes dont elle s'est donné pour tâche de rendre compte. Je tente ici de montrer en quoi diverses approches pragmatiques ont néanmoins aménagé un espace pour traiter ce dernier, et prends appui sur la pragmatique cognitive pour défendre l'hypothèse qu'une telle pragmatique du sens verbal fournit des outils exploitables pour une pragmatique de la persuasion/conviction, par essence perlocutoire. Les avantages méthodologiques de cette démarche, à même d'intéresser les rhétoriciens, sont également discutés.

INDEX

Mots-clés: acte perlocutoire, contraintes interprétatives, pragmatique, rhétorique

Keywords: interpretative constraints, perlocutionary act, pragmatics, rhetoric

\section{AUTHOR}

STEVE OSWALD

Université de Fribourg 